\title{
Abordagem familiar: hereditariedade do etilismo em uma família
}

\author{
Family approach: heredity of alcoholism in a family
}

Enfoque familiar: herencia del alcoholismo en una familia

Jaderson Vinicius Cardoso de Souza ${ }^{1 *}$, Brunna Thaís Costa1, Maíla Lorena de Oliveira Sarmento1, Juliana Almeida Rocha', Wártineê Dias Miranda Lacerda', Edmar Rocha Almeida ${ }^{1}$, Andra Aparecida da Silva Dionízio ${ }^{1}$, Carlos Alberto Quintão Rodrigues'1, Rafael Márcio dos Santos Souza'.

\section{RESUMO}

Objetivo: Descrever uma ação de intervenção familiar para redução dos danos relacionados ao alcoolismo. Detalhamentos de caso: Trata-se de um estudo de caso com abordagem metodológica qualitativa, realizado através de atendimentos individuais e domiciliares, aplicação das ferramentas de acesso: genograma, ecomapa, ciclo de vida, FIRO, P.R.A.C.T.I.C.E, conferência familiar e desenvolvimento de projeto terapêutico singular (PTS) elaborado pela equipe multiprofissional. O paciente Renato é um adulto jovem que apresenta desestruturação familiar e comprometimento da saúde associado ao etilismo crônico. O emprego das ferramentas possibilitou elaborar um plano de cuidado com visão holística abordando o etilismo. Além disso, identificou-se outros agravos como desemprego, tabagismo e agressões. Foram propostas intervenções caracterizadas por instituição de tratamento medicamentoso, ações visando melhoria do convívio, obtenção de novas fontes de renda, lazer e relacionamento social, além do fortalecimento do vínculo da rede de apoio com a família. Considerações finais: A abordagem familiar favoreceu a proximidade da família com a equipe de saúde, permitindo condutas pertinentes à condição disfuncional evidenciada.

Palavras-chave: Relações familiares, Estratégia saúde da família, Alcoolismo, Violência doméstica.

\section{ABSTRACT}

Objective: To describe a family intervention action to reduce harm related to alcoholism. Case Details: This is a case study with a qualitative methodological approach, carried out through individual and home care, application of access tools: genogram, ecomap, life cycle, FIRO, PRACTICE, family conference and therapeutic project development (PTS) prepared by the multiprofessional team. The patient Renato is a young adult who has family dysfunction and impaired health associated with chronic alcoholism. The use of tools made it possible to elaborate a care plan with a holistic view addressing alcoholism. In addition, other problems such as unemployment, smoking and aggression were identified. Interventions were proposed, characterized by the institution of drug treatment, actions aimed at improving social interaction, obtaining new sources of income, leisure and social relationships, in addition to strenghening the bond of the support network with the family. Final considerations: The family approach favored the family's proximity to the health team, allowing behaviors pertinent to the dysfunctional condition evidenced.

Keywords: Family relations, Family health strategy, Alcoholism, Domestic violence.

\section{RESUMEN}

Objetivo: Describir una acción de intervención familiar para reducir el daño relacionado con el alcoholismo. Detalles del caso: Este es un estudio de caso con un enfoque metodológico cualitativo, realizado a través de la atención individual y domiciliaria, aplicación de herramientas de acceso: genograma, ecomapa, ciclo de vida, FIRO, PRÁCTICA, conferencia familiar y desarrollo de proyectos terapéuticos. (PTS) preparado por el equipo multiprofesional. El paciente Renato es un adulto joven que tiene disfunción familiar y problemas de salud asociados con el alcoholismo crónico. El uso de herramientas permitió elaborar un plan de atención con

1 Universidade Estadual de Montes Claros (UNIMONTES), Montes Claros - MG.

*E-mail: jadersonvinicius@yahoo.com.br

SUBMETIDO EM: $8 / 2020$

ACEITO EM: 9/2020

PUBLICADO EM: 11/2020

REAS/EJCH | Vol.12(11) | e4905 | DOI: https://doi.org/10.25248/reas.e4905.2020 Página 1 de 7 
una visión holística que abordara el alcoholismo. Además, se identificaron otros problemas como el desempleo, el tabaquismo y la agresión. Se propusieron intervenciones, caracterizadas por la institución del tratamiento de drogas, acciones dirigidas a mejorar la interacción social, obtener nuevas fuentes de ingresos, ocio y relaciones sociales, además de fortalecer el vínculo de la red de apoyo con la familia. Consideraciones finales: el enfoque familiar favoreció la proximidad de la familia al equipo de salud, permitiendo comportamientos relevantes para la condición disfuncional evidenciada.

Palabras clave: Relaciones familiares, Estrategia de salud familiar, Alcoholismo, Violencia doméstica.

\section{INTRODUÇÃO}

A Família pode ser definida como conjunto de pessoas interligadas por laços emotivos ou consanguíneos que compartilham uma história em comum, por dependência doméstica, convivência e que comumente residem no mesmo lar. Como forma de cuidado à família tem-se a Estratégia Saúde da Família (ESF) que foi implementada pelo Ministério da Saúde para reorganização da Atenção Primária à Saúde (APS), sendo considerada uma alternativa importante para o alcance dos escopos de universalização, equidade e integralidade (BRANTE ARS, et al., 2016, BATISTA RS, et al., 2015).Com isso, a ESF se norteia em elementos para o desenvolvimento das práticas de saúde, com foco central no indivíduo/família, integralidade e disposição da atenção, rede assistencial, a atuação da comunidade e o exercício intersetorial (ARANTES LJ, et al., 2016).

A abordagem familiar é proposta por Starfield B (2002) como um dos atributos da APS. Assim é imprescindível que a ESF entenda os integrantes da família como um todo bem como seus agravos de saúde. Ao compreender os padrões familiares, inicia-se a realização de intervenções adequadas ao cenário social em que as famílias estão inseridas.

Para tanto, podem ser empregados instrumentos como: Genograma (estrutura e padrão das relações familiares), ecomapa (vínculos entre membros da família e comunidade), ciclo de vida familiar (estágios de desenvolvimento familiar), (FIRO) Fundamental Interpersonal Relations Orientations (compreender o funcionamento da família pela relação de afeto, escuta e poder), (P.R.A.C.T.I.C.E) Present Problem; Roles and Structure; Affect; Communication; Time in the Family life cycle; Illness in Family past and present; Coping with stress; Ecology (organização e posição da família diante dos problemas) e conferência familiar (compartilhamento de informações e sentimentos objetivando mudar rotinas de interação na família) (SANTOS JAD, et al., 2016).

No contexto do estudo da estrutura de uma família, identifica-se a inferência do abuso do álcool na disfuncionalidade do meio familiar, atingindo todos os membros, ao aumentar a violência doméstica, desacordo entre os familiares, divórcios, negligência infantil, chegando a acarretar também dificuldades financeiras (MALBERGIER A, et al., 2013). Nesse sentido objetivou-se descrever o caso de avaliação e intervenção familiar para redução dos danos causados pelo alcoolismo.

\section{DETALHAMENTO DE CASO}

Trata-se de um estudo de caso de abordagem metodológica qualitativa, desenvolvido entre outubro de 2019 a fevereiro de 2020 no território de uma equipe da ESF, área de atuação do Programa de Residência Multiprofissional em Saúde da Família e Comunidade da Universidade Estadual de Montes Claros, (UNIMONTES), em um município de Minas Gerais.

Foi submetido ao Comitê de Ética em Pesquisa da UNIMONTES, aprovado em 26 de outubro de 2016, conforme parecer no 1.792.192 e Certificado de Apresentação para Apreciação Ética 60676416.8.0000.5146, os membros da família assinaram o Termo de Consentimento Livre e Esclarecido, após consultado seu interesse em participar do estudo, garantindo sigilo das informações coletadas e desistência a qualquer momento. Foram utilizados nomes fictícios para descrição e confidencialidade do caso, resguardando assim o anonimato. 
Esta abordagem iniciou-se a partir da observação do quadro de etilismo de um jovem que encetou precocemente o uso do álcool, acarretando inúmeros impasses na organização e convívio de sua família e danos à sua saúde. Para sua execução foram realizados atendimentos individuais e domiciliares, a fim de compreender a estrutura familiar, o que permitiu a aplicação das ferramentas de acesso à família.

O genograma consiste em um diagrama expressivo que proporciona uma visão ampla dos padrões e convivência familiar que envolvem o processo saúde-doença. As representações gráficas das relações do cidadão envolvido foi construída a partir do aplicativo Álbum de Família do Núcleo de Educação em Saúde Coletiva da Faculdade de Medicina da Universidade Federal de Minas Gerais (Figura 1) (BORGES CD, 2015).

A família é composta por 16 integrantes destes, cinco residem juntos formando o núcleo familiar, as condições da moradia são básicas, a casa é ampla e a limpeza do ambiente é adequada. Renato, 29 anos, desempregado, divorciado, hipertenso, tabagista, etilista crônico há 14 anos, com diagnóstico de hepatite alcoólica aos 22 anos, possui histórico de internação hospitalar em decorrência de quadro de pancreatite aguda há um ano e diversos atendimentos em serviços de saúde devido uso abusivo de álcool.

Renato é progênie do segundo casamento de Joelma 59 anos, tabagista, asmática e hipertensa com João etilista crônico falecido, dessa relação tiveram outro filho, Henrique 31 anos, mecânico, casado. Joelma atualmente é casada com Marcio 70 anos, alcoólatra, diabético, tabagista, esquizofrênico em acompanhamento contínuo no Centro de Atenção Psicossocial (CAPS), desta relação gerou uma filha, Hellen de 22 anos, no momento em acompanhamento na ESF devido quadro de ansiedade, ela é mãe de Davi de dois anos. Renato não tem relação de proximidade com Ronaldo e Gisele irmã de Renato.

Segundo relato de Henrique, o senhor João foi encontrado alguns dias após o seu falecimento, ocasionado pelo excesso de consumo alcoólico, o que provoca uma dor muito grande em ver seu irmão Renato seguindo os passos do pai, situação que caracteriza hereditariedade do alcoolismo no seio familiar. Renato é pai de Carlos de 11 anos que reside com a mãe, possui vínculo distante e os períodos de convivência são marcados por episódios de embriaguez. Segundo Joelma o filho tenta se aproximar do pai, mas o alcoolismo se tornou uma barreira.

Figura 1 - Genograma familiar de Renato, Taiobeiras - MG, 2020.

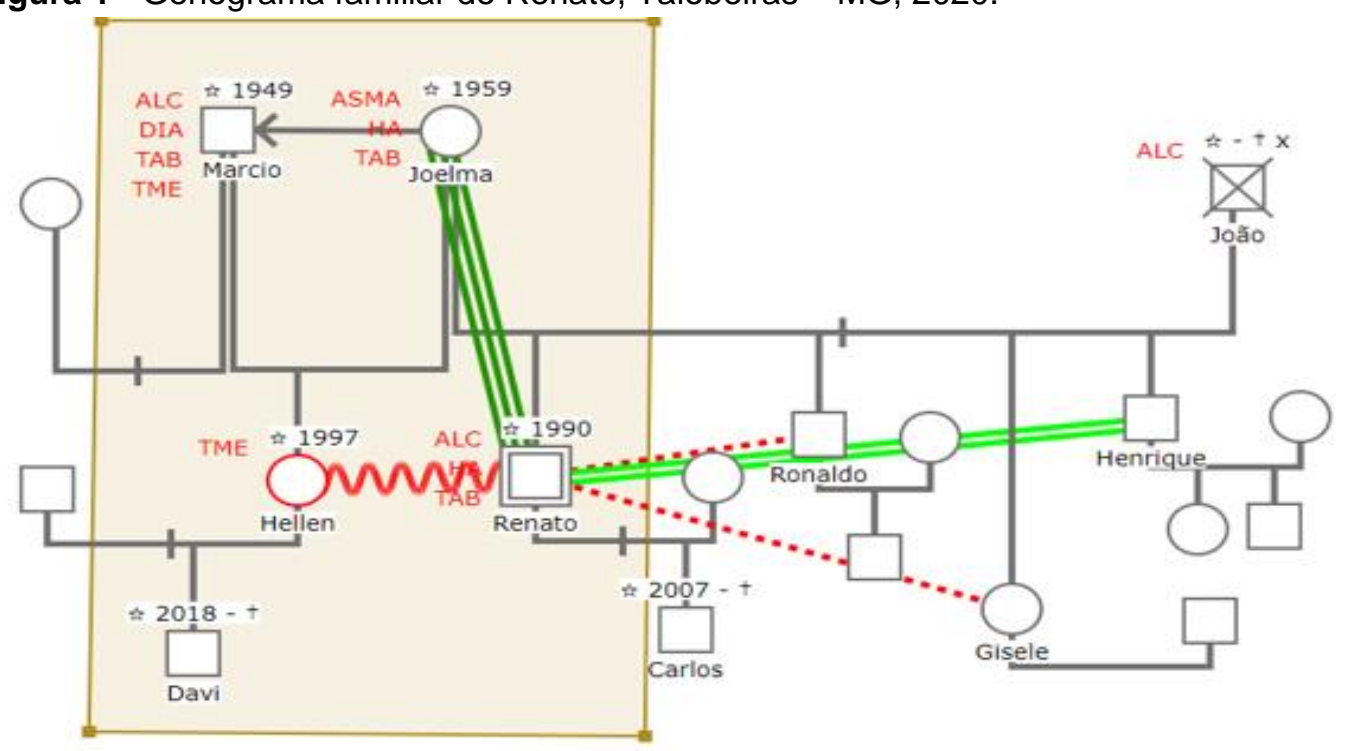

LEGENDA

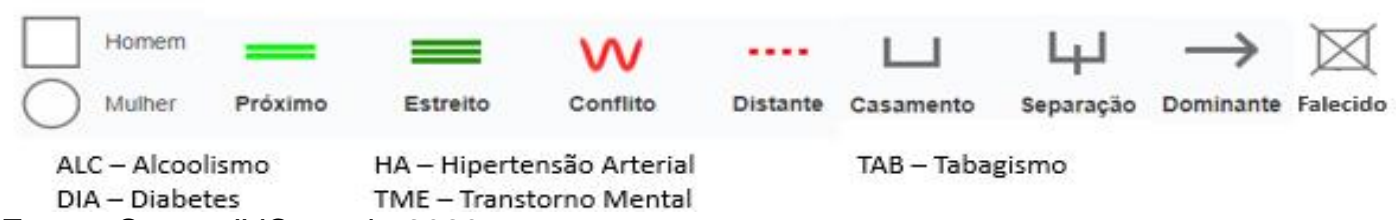

Fonte: Souza JVC, et al., 2020. 
Complementando o genograma, o ecomapa auxiliou no entendimento das relações entre família com meio social em que está inserida, permitindo ainda conhecer todo suporte externo como igreja, vizinhança, amigos e estabelecimentos de saúde. Os dados obtidos a partir da aplicação do ecomapa evidenciou relação forte com a ESF, vínculo inexistente com a igreja, vizinhos e trabalho. Renato mantém forte ligação com os amigos que incentivam o alcoolismo, o que configura um gatilho para manutenção da dependência (Figura 2).

Tendo em vista as relações frágeis apresentadas pela família, há necessidade de fortalecer a rede de apoio, uma vez que ela se caracteriza pela construção de vínculo próximo e intensidade afetiva. De forma a complementar o vínculo com a ESF, foram convidados a participar dos grupos em educação em saúde, reuniões com a população e acolhimento como forma de aproximação da equipe e cuidado em saúde (COSTA PHA, 2016; SOUZA IP, 2016).

Figura 2 - Ecomapa familiar de Renato, Taiobeiras - MG, 2020.

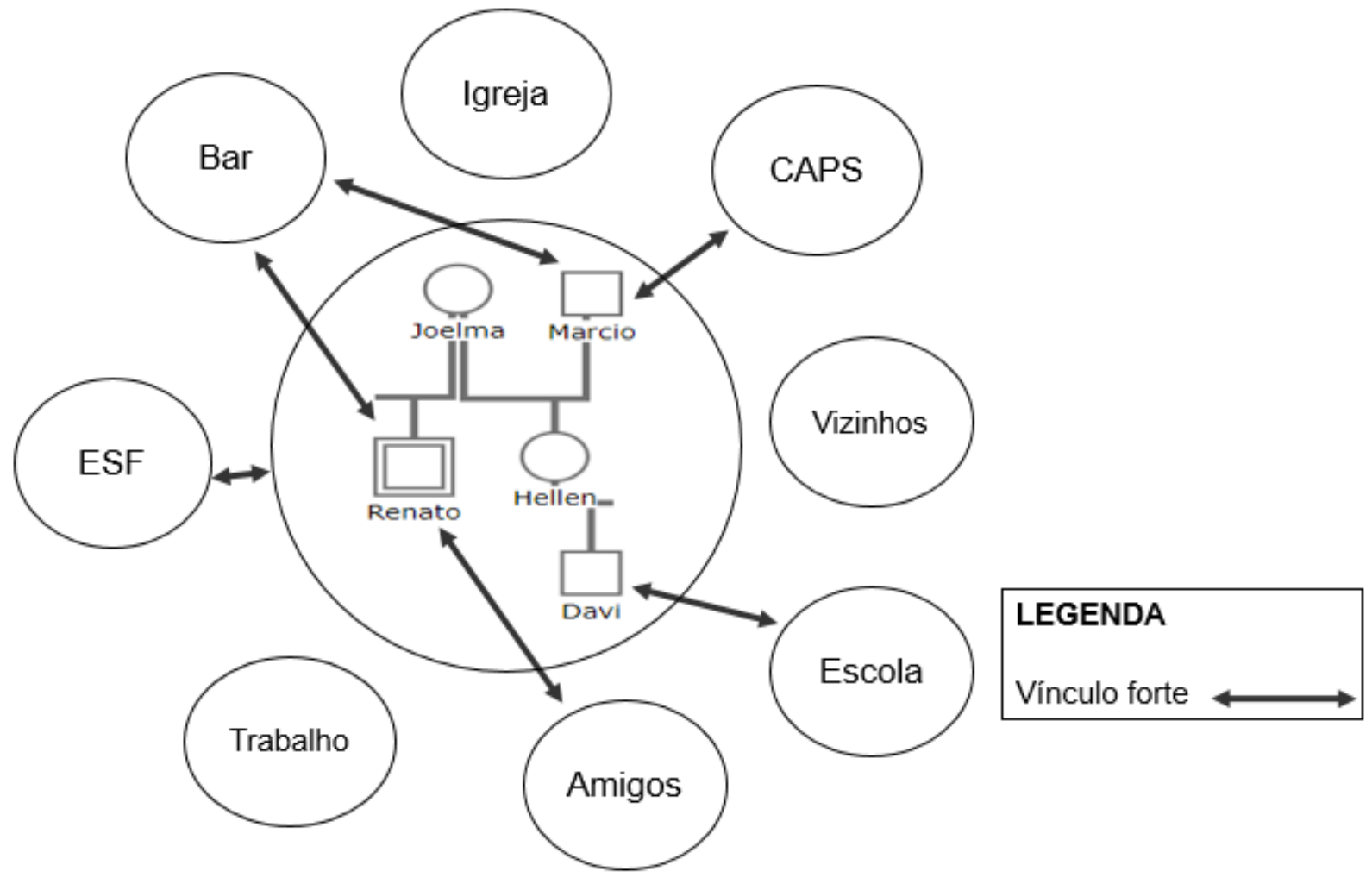

Fonte: Souza JVC, et al., 2020.

O ciclo de vida representa etapas que as famílias passam e os desafios/tarefas a cumprir em cada uma delas. Conhecimento pertinente, pois se mostra útil nas próximas adversidades que acontecerão conforme desenvolvimento do ciclo de vida constatado (FELICIO LFF, 2018). Os membros desse estudo vivem mais de um ciclo concomitantemente: famílias com filhos pequenos e aposentadoria, nota-se despreparo em relação aos ajustes da convivência e espaço.

Diante do ciclo de vida familiar identificado foi agendado atendimento de puericultura reforçando o cuidado com a saúde infantil e abordagem da alimentação saudável, vacinação, educação, saúde bucal, prevenção de acidentes, higiene e a necessidade de um ambiente familiar harmonioso. Essas ações foram ofertadas a partir da necessidade de articular e inserir a família nas práticas educativas, visando o desenvolvimento e crescimento saudável da criança, bem como a discussão do processo de envelhecimento como um fator esperado e natural (CHAPADEIRO CA, et al., 2013).

O instrumento FIRO foi empregado como forma de avaliar as dimensões de inclusão, controle e intimidade (LACERDA MKS, et al., 2017). Renato sente-se excluído nas relações intrafamiliares. A família demonstrou vínculo frágil, sendo que os membros possuem dificuldade na resolução dos conflitos. 
O controle refere-se às interações do exercício de poder dentro da família. Podem ser identificadas situações de controles: dominante, reativo e colaborativo, Joelma exerce manejo dominante sobre os demais membros da família. O filho Henrique e o padrasto Marcio estabelecem um mando colaborativo, ao passo que Renato e Hellen apresentam-se reativos, sendo contrário ao que é ansiado pela família. Já a intimidade relaciona às interações correlatas às trocas interpessoais, ao modo de compartilhar sentimentos, ao desenvolvimento de atitudes de aproximação ou de distanciamento, às vulnerabilidades e às fortalezas. Os familiares demonstram ter pouca intimidade entre si, sendo o ambiente um local de precária interação e comunicação limitada, demonstrações de afeto não foram identificados.

Com base nos resultados obtidos por meio da ferramenta FIRO, foi realizado pela psicóloga do Núcleo Ampliado de Saúde da Família - Atenção Básica, (NASF-AB) intervenção psicossocial visando melhoria das relações interpessoais. O domínio predominante de Joelma revelou a necessidade de aceitação e preparação para uma possível mudança dos papéis dentro da família, levando em consideração a senilidade e comprometimento atual de sua saúde.

O P.R.A.C.T.I.C.E contribui com a avaliação familiar, resolução de problemas e favorece nas intervenções. A ferramenta disponibiliza noções sobre a organização e colocação da família com foco principal no problema identificado, facilitando intervenções pertinentes a adversidade. (BRANTE ARS, et al., 2016). Observe na tabela a seguir a descrição da ferramenta empregada na família (Quadro 1).

\begin{tabular}{|c|c|c|}
\hline $\mathrm{P}$ & Problem (Problema) & Alcoolismo do Renato é responsável pelos conflitos existentes. \\
\hline $\mathrm{R}$ & $\begin{array}{l}\text { Roles and structure (papeis } \\
\text { e estrutura) }\end{array}$ & $\begin{array}{l}\text { Joelma assume as funções de mãe, esposa e avó, além da execução das } \\
\text { tarefas domésticas juntamente com a filha Hellen. Já Marcio apesar de ser } \\
\text { provedor financeiro, desempenha uma função passiva nas decisões } \\
\text { familiares. Renato não auxilia na manutenção do lar. }\end{array}$ \\
\hline A & Affect (afeto) & $\begin{array}{c}\text { Renato possui proximidade com Henrique, contudo tem vínculo distante } \\
\text { com o filho e convivência conflituosa com a Hellen, em alguns momentos } \\
\text { houve relatos de agressões física e verbal. A família demonstra relação de } \\
\text { respeito com Marcio. }\end{array}$ \\
\hline C & $\begin{array}{l}\text { Communication } \\
\text { (comunicação) }\end{array}$ & $\begin{array}{l}\text { Nota-se que é deficiente Renato demonstra dificuldade em se comunicar } \\
\text { sendo passivo às questões da família, já Joelma consegue estabelecer } \\
\text { diálogo com os membros. }\end{array}$ \\
\hline $\mathrm{T}$ & $\begin{array}{c}\text { Time in life (tempo no ciclo } \\
\text { de vida) }\end{array}$ & Famílias com filhos pequenos e aposentadoria. \\
\hline I & $\begin{array}{l}\text { IIIness in family past and } \\
\text { present (doenças na } \\
\text { família) }\end{array}$ & $\begin{array}{l}\text { Renato Hipertenso, etilista e tabagista. Joelma asmática, hipertensa e } \\
\text { tabagista. Marcio Etilista, diabético, tabagista e esquizofrênico. Hellen } \\
\text { ansiedade. Ambos encaram essas doenças com naturalidade. }\end{array}$ \\
\hline C & $\begin{array}{l}\text { Coping with stress (lidar } \\
\text { com o estresse) }\end{array}$ & $\begin{array}{l}\text { Os membros da família possuem dificuldades em lidar de forma resolutiva, } \\
\text { tendendo ao isolamento e falta de esperanças nas mudanças. }\end{array}$ \\
\hline $\mathrm{E}$ & $\begin{array}{l}\text { Environment/ecology (meio } \\
\text { ambiente) }\end{array}$ & $\begin{array}{c}\text { Os moradores possuem vínculo satisfatório com a ESF e buscam auxílio } \\
\text { sempre que necessário. }\end{array}$ \\
\hline
\end{tabular}

Fonte: Souza JVC, et al., 2020.

A ferramenta P.R.A.T.I.C.E possibilitou a equipe identificar diversos problemas de convívio e diálogo entre os membros. É necessário que todos os integrantes da família tenham conhecimento da importância do seu desempenho adequado ao papel familiar, indispensabilidade em manter uma posição flexível que possibilite conversa amigável entre os familiares.

Com a aplicação dessas ferramentas e visando o contexto em que a família está inserida, foi desenvolvido um Plano Terapêutico Singular (PTS) caracterizado pela percepção interdisciplinar de várias especialidades e de distintas profissões, com condutas terapêuticas que vinculam entre si (FERREIRA SO, et al., 2015).

O PTS foi desenvolvido de forma multiprofissional, proposto aos integrantes etilistas a buscarem grupos de apoio existentes no município como os Alcoólicos Anônimos que se reúnem para alcançar e manter a sobriedade e abstinência total de ingestão de bebidas alcoólicas. Quanto a Joelma que além de tabagista é asmática, foi esclarecido os riscos e agravos provenientes do hábito tabágico. 
Ainda dentro do PTS, considerando a renda familiar e desemprego, foi informado e encaminhado sobre os serviços prestados pelo Centro de Referência de Assistência Social, que incluem oficinas profissionalizantes com o objetivo de contribuir com a renda familiar e ocupação do tempo ocioso, podendo ser utilizado como forma de laser e socialização. Atualmente Renato e sua família encontram-se sobre acompanhamento supervisionado pela ESF, onde foi observado diminuição do consumo de álcool após o início do tratamento, entretanto Joelma relata persistência de violência doméstica visto que não houve o êxito esperado através da cessação total do alcoolismo.

A conferência familiar foi realizada com a presença de Renato, Joelma, Henrique, Marcio e Hellen. A equipe foi acolhida pelos usuários em seu domicílio e inicialmente foi exposto os principais problemas identificados durante a aplicação das ferramentas, dos quais: conflito familiar decorrente do etilismo crônico tendo como resultado episódios de violência doméstica. A família foi ouvida e todos os membros presentes confirmaram que os atos de violência entre Hellen e Renato ocorrem após o consumo de álcool, que tornam o comportamento de Renato agressivo contra Hellen que reage através de agressões físicas. Os ataques são constantes e gera desconforto, Joelma temendo pela vida dos filhos, diversas vezes busca minimizar o conflito entre os dois irmãos.

Durante a conferência houve um momento de comoção, após Henrique se recordar do pai biológico, comparando a trajetória de vida e o consumo excessivo de álcool e tabaco do mesmo com o Renato, reforçando a ideia de hereditariedade etilista na família.

Foram elencadas três propostas de intervenção direcionadas ao paciente em estudo: internação por 12 meses em uma clínica de reabilitação regional para dependentes químicos oferecido gratuitamente por instituição filantrópica; tratamento no CAPS ou acompanhamento na unidade de saúde.

Ao final Renato demonstrou interesse em iniciar tratamento para cessação do vício, contudo naquele momento não conseguiu escolher o método terapêutico, mas se comprometeu a procurar a unidade de saúde posteriormente. Renato compareceu à unidade sete dias após a conferência familiar para iniciar o tratamento, foi realizado atendimento compartilhado com abordagem multiprofissional, disponibilização de orientações e instituição de propedêutica.

\section{DISCUSSÃO}

Com base no estudo e aplicabilidade das ferramentas de abordagem familiar, foi possível realizar uma provável associação do etilismo do paciente Renato ao histórico de dependência do pai. Em um estudo feito por Moraes SJR e Barroco SMS (2016), descreve o alcoolismo como doença crônica, com associação genética, psicossocial e ambiental influenciando seu desenvolvimento e manifestações. O estudo relaciona determinantes do alcoolismo em categorias (biológicas, psicológicas/comportamentais e ambientais) e elementos como: sexo, idade, fatores genéticos e marcadores orgânicos como enzimas e taxas sanguíneas.

Corroborando com os achados, Oliveira, et al. (2019) ressalta a conceituação do etilismo como uma patologia decorrente de causas biológicas e genéticas, esta caracterização aproxima-se do conceito atual que se tem sobre a dependência, os quais enfatizam história familiar, aspectos da personalidade, padrões de consumo e psicopatologias.

A aplicação da ferramenta ciclo de vida evidenciou a etapa: famílias com filhos pequenos e aposentadoria. Baseando-se na perspectiva de hereditariedade e incidência do alcoolismo entre jovens e adolescentes, determinantes ambientais devem ser evitados como forma preventiva ao etilismo infantil. O que corrobora com o estudo de Silva KR e GOMES FGC (2019) onde evidencia que mais da metade das famílias de dependentes químicos possuem um outro membro que apresenta problemas com o uso de álcool ou drogas, diante destes indícios há relevante associação da dependência como um hábito adquirido, sob influência familiar.

Diante dos fatos apresentados, a família demostra relacionamento conflituoso entre os membros o que agrava a resolução e entendimento dos conflitos identificados. De acordo com Almeida CP (2020) é no círculo familiar que o indivíduo alcança requisitos para se desenvolver por meio de apoio afetivo e emocional, ambiente marcado por relações de agressividade e conflitos prejudicam o desenvolvimento psicológico e ativo do contexto familiar. 
Dessa forma a conferência familiar realizada, identificou padrões de relacionamento precário entre os membros com variados sentimentos e atitudes atípicas de alguns integrantes, o alcoolismo por exemplo. Moraes SJR e Barroco SMS (2016) apontam que, as relações familiares apresentam condições de proteção ou risco para o etilismo, baseado na característica da família. Considerando características importantes como a presença ou não de respeito e transigência entre os membros, nível de conhecimento dos integrantes sobre os prejuízos do álcool, uso no meio familiar e hábitos rotineiros referentes a esse assunto.

A abordagem familiar viabilizou a realização de plano de cuidado e valorização dos membros da família, com foco nas intervenções em combate ao etilismo e comprovação de hereditariedade como possível influencia no etilismo. Iniciativa que permitiu interação com o lar, obtendo confiabilidade para as informações repassadas mediante observações, análise do convívio e demandas apresentadas.

Com isso, utilizando as ferramentas de acesso foi plausível conhecer o processo saúde/doença e as repercussões e os impactos do etilismo, agressões e o desemprego. Foi possível notar demandas, tormentos, suplícios e potenciais agravos que antes seriam ignorados, e com isso apresentado uma forma de superação dos problemas em uma prática de atenção à saúde voltada na família. Apesar do trabalho da equipe, entre orientações, propostas de intervenções e encaminhamentos, o indivíduo tem autonomia e responsabilidade sobre sua saúde, o que limita assim o trabalho da ESF.

\section{AGRADECIMENTOS}

Ao Programa de Residência Multiprofissional em Saúde da Família e Comunidade da Universidade Estadual de Montes Claros - MG.

\section{REFERÊNCIAS}

1. ALMEIDA CP, et al. Abordagem familiar: estudo de caso de uma família no município de Taiobeiras, Minas Gerais, Brasil. Revista Eletrônica Acervo Saúde, 2020; 51: e3545.

2. ARANTES LJ, et al. Contribuições e desafios da estratégia saúde da família na atenção primária à saúde no Brasil: revisão da literatura. Ciência \& Saúde Coletiva, 2016; 21: 1499-1510.

3. BATISTA RS, et al. (Bio) ética e estratégia saúde da família: mapeando problemas. Saúde e Sociedade, 2015; 24: 113-128.

4. BORGES CD, et al. Genograma e atenção básica à saúde: em busca da integralidade. Revista Psicologia e Saúde, 2015; 7 (2): 133-141.

5. BRANTE ARSD, et al. Abordagem familiar: aplicação de ferramentas a uma família do município de Montes Claros/MG. Revista Brasileira de Medicina de Família e Comunidade, 2016; 11(38): 1-9.

6. CHAPADEIRO CA, et al. A família como foco da atenção primária à saúde. Nescon/UFMG, 2013; 100.

7. COSTA PHA, et al. O Ecomapa como ferramenta na formação para o trabalho em rede no campo de álcool e outras drogas. Revista Pesquisas e Práticas Psicossociais, 2016; 11 (3): 669-681.

8. FELICIO LFF, et al. Abordagem familiar no cuidado ao paciente com esquizofrenia: relato de caso conduzido na atenção primária à saúde. Revista Eletrônica Acervo Saúde, 2018; 10 (3): 1621-1627.

9. FERREIRA SO, et al. Construção de um projeto terapêutico singular durante visita domiciliar: relato de experiência. Cidadania em Ação: Revista de Extensão e Cultura, 2015; 9(1): 120-129.

10. LACERDA MKS, et al. Ferramentas de abordagem familiar: estudo de uma família cadastrada em uma equipe de estratégia saúde da família em Montes Claros - MG. Revista de Iniciação Científica da Universidade Vale do Rio Verde, 2017; 7(1).

11. MALBERGIER A, et al. Uso de substâncias na adolescência e problemas familiares. Cadernos de Saúde Pública, 2013; 28(4): 678-688.

12. MORAES SJR, BARROCO SMS. Concepções do alcoolismo na atualidade: pesquisas hegemônicas, avanços e contradições. Psicologia: Teoria e Pesquisa, 2016; 32 (1).

13. OLIVEIRA et al. A construção histórica do estigma sobre o conceito de dependência de alcool. ID online - Revista Multidisciplinar e de Psicologia, 2019; 13 (44): 253-275.

14. SANTOS JAD, et al. Ferramenta de abordagem familiar na atenção básica: um relato de caso. Journal of the Health Sciences Institute. 2016; 34(4): 249-52.

15. SILVA KR, GOMES FGC. Dependência química: resultantes do uso abusivo de substâncias psicoativas, Revista UNINGÁ, 2019; 56 (1): 186-195.

16. SOUZA IP, et al. Genograma e ecomapa como ferramentas para compreensão do cuidado familiar no adoecimento crônico de jovem. Texto e Contexto Enfermagem, 2016; 4: 1-10.

17. STARFIELD B. Atenção primária: equilíbrio entre necessidades de saúde, serviços e tecnologia. Brasília: Organização das Nações Unidas para a Educação, a Ciência e a Cultura (UNESCO), Ministério da Saúde, 2002; $726 p$. 